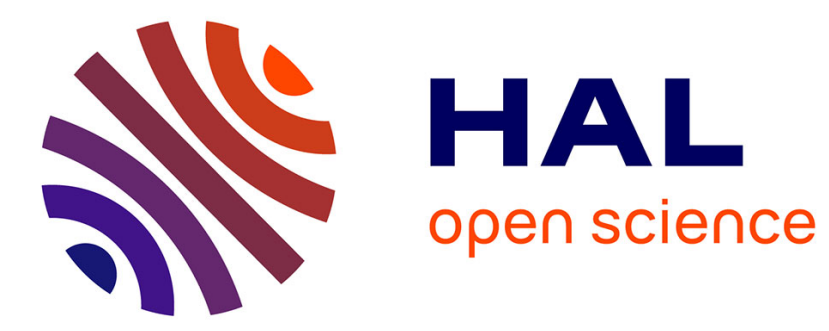

\title{
Detection and prevention from misbehaving intruders in vehicular networks
}

\author{
Hichem Sedjelmaci, Tarek Bouali, Sidi Mohammed Senouci
}

\section{To cite this version:}

Hichem Sedjelmaci, Tarek Bouali, Sidi Mohammed Senouci. Detection and prevention from misbehaving intruders in vehicular networks. 2014 IEEE Global Communications Conference, Dec 2014, Austin, United States. 10.1109/GLOCOM.2014.7036781 . hal-02557404

\author{
HAL Id: hal-02557404 \\ https://hal.science/hal-02557404
}

Submitted on 14 Feb 2022

HAL is a multi-disciplinary open access archive for the deposit and dissemination of scientific research documents, whether they are published or not. The documents may come from teaching and research institutions in France or abroad, or from public or private research centers.
L'archive ouverte pluridisciplinaire HAL, est destinée au dépôt et à la diffusion de documents scientifiques de niveau recherche, publiés ou non, émanant des établissements d'enseignement et de recherche français ou étrangers, des laboratoires publics ou privés.

\section{다)(1) $(5$}

Distributed under a Creative Commons Attribution - NonCommerciall 4.0 International 


\title{
Detection and Prevention From Misbehaving Intruders in Vehicular Networks
}

\author{
Hichem Sedjelmaci, Tarek Bouali and Sidi Mohammed Senouci \\ University of Bourgogne, DRIVE Lab \\ 49 Rue Mademoiselle Bourgeois, 58000, Nevers, France \\ \{Sid-Ahmed-Hichem.Sedjelmaci, tarek.bouali, Sidi-Mohammed.Senouci\}@u-bourgogne.fr
}

\begin{abstract}
In this paper, we design and implement a new intrusion detection and prevention schema for vehicular networks. It has the ability to detect and predict with a high accuracy a future malicious behavior of an attacker. This is unlike the current detection schemas, where there is no prevention technique since they aim to detect only current attackers that occur in the network. We used game theory concept to predict the future behavior of the monitored vehicle and categorize it into the appropriate list (White, White \&Gray, Gray, and Revocation_Black) according to its predicted attack severity. In this paper, our aim is to prevent from the most dangerous attack that targets a vehicular network, which is false alert's generation attack. Simulation results show that our intrusion detection and prevention schema exhibits a high detection rate and generates a low false positive rate. In addition, it requires a low overhead to achieve a high-level security.
\end{abstract}

Keywords- Vehicular networks; Intrusion detection and prevention; Game theory; Attacks

\section{INTRODUCTION}

Vehicle Ad hoc Networks (VANETs) are attracting much attention from both academia and industry. Indeed, vehicular networks are used to provide multiple services, which are categorized into two classes: infotainment and safety services. We can cite as safety services examples developed by ITEA2 CarCoDe project [1]: real-time traffic monitoring and management, and real-time fleet management. Therefore, securing such networks is very critical, especially for safety applications since they are attractive target due to the relevant information that vehicles can manage [2].

Intrusion Detection System (IDS) is the most reliable mechanism to protect the network against threats since it has the ability to detect attackers with a high accuracy and prevent network disruption, e.g. exhaust a resource of a legitimate node or disseminate a false alert. Existing detection schemas relay only on the current behavior of the monitored node to compute its trust level (TL) and then take the final decision to categorize it at normal or malicious [2][4][5]. In this paper we are dealing with the malicious behavior's prediction and hence propose a reliable intrusion detection and prevention schema that has the ability to detect and especially prevent the malicious vehicle to attack. To the best of our knowledge, this is the first work that uses malicious behavior's prediction in vehicular networks. Our schema relies on game theory to predict the malicious behavior of an attacker and categorizes the monitored vehicle into an appropriate list according to their future attack severity. We formulate the attack-defense problem as a Bayesian game formulation between the attacker and RSU, and the futures states' prediction of a suspected behavior is determined thanks to Bayesian Nash Equilibrium $(B N E)$. Furthermore, in this research, we focus to detect the most dangerous attack that could occur in VANET safety applications, which is a False alert's generation attack. The aim of such attack is to generate a false alert in order to create for example a traffic jam. Therefore, we propose a set of detection rules to identify such malicious behavior when it occurs.

According to our simulation results, we prove that our malicious behavior's prediction technique that relies on game theory has the ability to predict with a high accuracy a future malicious behavior of a monitored vehicle even when the number of vehicles is high (i.e. scalable). In addition, our detection and prevention schema requires a low overhead to detect and prevent the attackers' occurrence.

This document is structured as follows: In Section 2, we present some intrusion detection schemas for VANETs proposed in the current literature. In Section 3, we present the network architecture that we attempt to secure. In addition, we describe some relevant alert messages that can be an attractive target for the attacker, and provide the detection policy to detect them. In Section 4, our malicious behavior's prediction based on game theory is explained and Section 4 provides NS3 simulation results. Finally, conclusion and future work conclude this paper.

\section{RELATED WORK}

The intrusion detection system is a most reliable technique to detect malicious vehicles with a high accuracy [3]. However, the current detection schemas proposed in VANET $[2][4][5][6][7]$ rely on current behavior of monitored node to compute its trust level (TL) and take a final decision (i.e. normal or malicious). In [4], the authors propose a robust and 
flexible trust model based on Markov Chain. In their approach they compute the TL of a monitored vehicle according to the following behaviors: the forwarding task and the reliability of a message that it sent. In their simulation results, they prove that their trust model reacts well to disruptive behaviors. However, the major drawback of this work is that security metrics such as detection and false positive rate are not evaluated. In [2][8], the authors aim to build a secure clusterbased framework, i.e. elect the most trusted node as a clusterhead $(\mathrm{CH})$. Specifically, in our recent work [2], we design and implement an intrusion detection framework in a cluster-based topology that aims to elect the cluster-head according to the monitored nodes' TL, i.e. the node with the highest TL is elected as $\mathrm{CH}$. In the simulation results, denial of service $(D o S)$ attacks are detected with a high accuracy even when the number of nodes is high. However, the detection framework generates a certain amount of overhead since a learning algorithm method is used to model a normal behavior of a target node.

In all these detection schemas, there is no prevention technique since they aim to detect only current attackers in the network. They do not have the ability to predict future misbehaviors.

\section{BACKGROUND AND DETECTION POLICY}

In this section, first of all we describe the network architecture that we attempt to secure. Second, we present the attack that we aim to detect and the proposed corresponding detection rules.

\section{A. Network architecture}

The network architecture that we attempt to secure is illustrated in Fig 1. This architecture is similar to the one used in the literature [9][10] where they assume vehicle to vehicle (V2V) and vehicle to infrastructure (V2I) communications. We use a dissemination protocol to disseminate an alert message to alert other vehicles when an event is detected. We note that, the forwarder nodes are selected according to their capability to provide a higher progress towards the destination (i.e. geographic area or RSU) [10]. In this research, we focus on three alerts' categories: Post Crash Notification (PCN), Road Hazard Condition Notification (RHCN) and Stopped/Slow Vehicle Advisor (SVA).

To identify a malicious node, each vehicle has the ability to monitor its neighbors and is called in the following "monitoring vehicle". However, when a malicious behavior is detected, the monitoring vehicle sends its feedback (i.e. monitoring list) to the RSU as explained bellow and illustrated in Fig 1. We assume that RSUs are connected to the application server through wired communication using a Transport Layer Security (TLS) protocol. This later aims to provide authentication and encryption in order to transmit sensitive data [11].

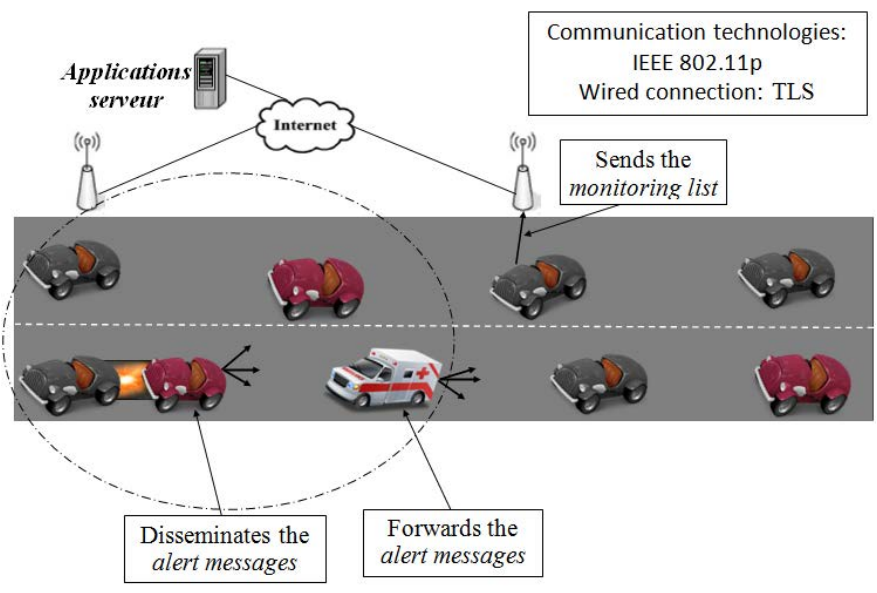

Fig. 1 Network architecture for VANET

\section{B. Attack model \& detection policy}

Alerts messages are important to ensure the safety of vehicles and persons on the road. However, a malicious vehicle could disseminate a false alert for its own purpose in order for instance to clear the road for itself or create a traffic jam in the road. Therefore, our aim in this paper is to detect this kind of malicious vehicles and secure the network from the following relevant false alerts: (i) Post Crash Notification (PCN): informs the vehicles that there is an accident ahead on the same roadway [5], (ii) Road Hazard Condition Notification (RHCN): warns vehicles about road conditions such as ice or unwanted debris on the road [5], and (iii) Stopped/Slow Vehicle Advisor (SVA): A low or stopped vehicle broadcasts warning messages to approaching vehicle while it is slow/stopped [5].

To detect the malicious vehicle with a high accuracy, we apply a mutual monitoring concept, i.e. each vehicle monitors its neighbors on a promiscuous mode. This later means that, the monitoring vehicle can hear all the packets that circulate with its radio range. The monitoring process aims to apply a set of rules to model a normal behavior of a vehicle after the message alert is raised, which are explained as follows:

When a vehicle disseminates an alert message about an event, the vehicles that are located within its radio range monitor its behavior to detect if this alert is correct. According to the authors in [5], when a node generates one of these alerts cited above, its speed should decrease and it should change lane. Hence, the monitoring vehicle analyzes the speed/lane of a monitored vehicle at both the moment and after the alert generation. As illustrated in Fig 2 (a), the vehicle $v m$ (attacker) sends periodically to its neighbor $v 1$ Beacon messages that include its position coordinates $\left(x_{\text {beacon }_{v m}}, y_{\text {beacon }_{v m}}\right)$, time when the message was generated $\left(t_{\text {beacon }}\right)$ and its current speed ( $\left.v m_{\text {speed_beacon }}\right)$. Furthermore, when the alert message is sent by $v m$, it includes information about the alert type (PCN, RHCN or SVA), its new position coordinates $\left(x_{\text {alert }_{v m}}, y_{\text {alert }_{v m}}\right)$, time when the alert was generated $\left(t_{\text {alert }}\right)$ and its speed $\left(v m_{\text {speed_alert }}\right)$. In this case, $v 1$ considered in this example as the monitoring node, computes the speed traveled between the alert generation and a subsequent beacon message as shown in equation (1). 
Speed $_{v m}=\frac{\mathrm{d}}{t_{\text {beacon }}-t_{\text {alert }}}$
$\mathrm{d}=\sqrt{\left(x_{\text {beacon }_{v m}}-\text { alert }_{v m}\right)^{2}+\left(y_{\text {beacon }_{v m}}-y_{\text {alert }_{v m}}\right)^{2}}$

When the formula (2) does not hold and the monitored vehicle does not change lane, $v m$ will be detected as a node that generates a false alert.

$$
\text { V is normal if }\left\{\begin{array}{c}
\text { Speed }_{V} \leq v_{\text {speed_alert }} \\
\text { and } \\
v_{\text {speed_beacon }}<v_{\text {speed_alert }}
\end{array}\right.
$$

As illustrated in Fig 2 (b), when a malicious behavior is detected, the monitoring vehicle $(v 1)$ disseminates a monitoring_message to inform its legitimate neighbors. This message includes: the source's identity $(v 1)$, the malicious vehicle's identity $(\mathrm{vm})$ and detection time. Furthermore, as shown in Fig 2 (b), $v l^{\prime} s$ neighbors monitor its behavior to verify if its speed has decreased and lane has changed. In case, when a false alert claimed by $v 1$ is correct, the neighbors store in their monitoring list the identity of the malicious vehicle and detection time. Otherwise, $v_{1}$ is designed as a node that disseminates false information and it will be stored in a monitoring list. At the end, this list will be forwarded to the nearest RSU as illustrated in Fig 1. However, in case when $\mathrm{RSU}$ is out of range, a store and forward mechanism is launched.
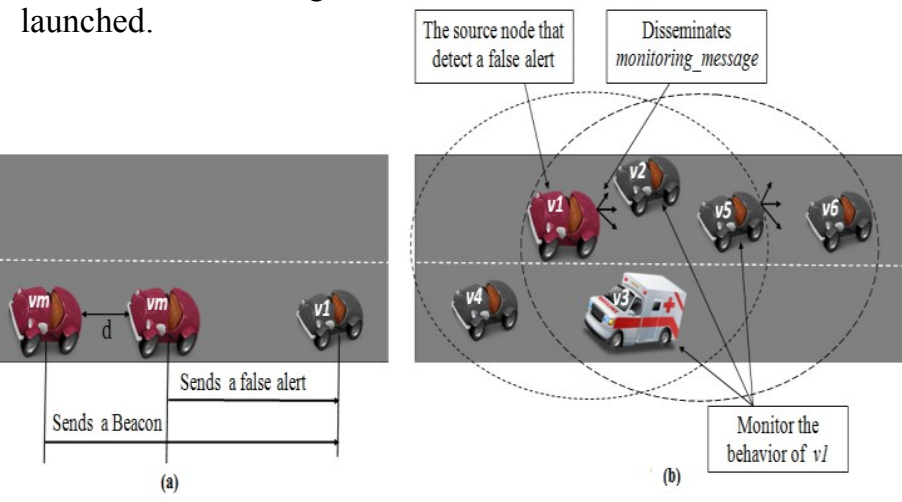

Fig. 2 Attack detection's scenario: (a) monitoring the target node's behavior, (b) dissemination of malicious vehicle's identity \& surveillance of the monitoring vehicle's behavior.

The RSU computes the malicious probability $(M P)$ related to $v_{j}$ during a detection period $\Delta \mathrm{t}$ as shown in equation (3). Afterward, each RSU exchanges between each other the monitoring lists. Each list includes the malicious vehicles' identities, time they were detected, RSU identity where they were attached and their $M P$ value. We note that, $\Delta \mathrm{t}$ was determined in our simulation experiments (see Table II).

$$
M P(\Delta \mathrm{t})=\frac{\sum_{i=1}^{n} R_{i} \cdot n b_{-} \text {detection }_{j}}{m} \quad \text { (3), and } 0 \leq M P \leq 1
$$

where, $n b_{-}$detection $_{j}$ (equal to $\mathrm{n}$ ) is the rate of monitoring vehicles that detect a vehicle $v_{j}$ as an attacker. $\mathrm{R}$ is the reliability detection of the monitoring vehicles, which depends on their detection history. In case, when monitoring vehicles provide correct detections during their passage through RSU range, $\sum_{i=1}^{n} R_{i}$ is equal to 1 , otherwise $\sum_{i=1}^{n} R_{i}$ tend to 0 when the vehicles persist to send a false detection. In our recent work [2], we explain how to compute $\mathrm{R}$ by using a vote mechanism.

and $m$ is the number of $v_{j}$ 's neighbors during a detection period $\Delta \mathrm{t}$.

Furthermore, with the help of game theory, the RSU predicts the future malicious behavior of a monitored vehicle and takes the final decision about its categorization. In the following, we explain our malicious behavior's prediction concept that relies on a series of game interaction between the monitored vehicle and the RSU.

\section{Proposed Malicious Behavior's Prediction Based ON GAME THEORY}

In this section, we describe our intrusion detection and decision performed between vehicles and RSU. First of all, we provide the payoff matrix of the game related to the monitored vehicle and RSU players and define a set of strategies that could occur between players. Afterwards, based on these strategies and with the help of Nash equilibrium concept, we predict the malicious behavior of an attacker in future states. Finally, according to this malicious behavior's prediction approach, we categorize the malicious vehicle according to its future severity's attack into an appropriate list.

\section{A. Game formulation}

In our approach, we consider two players: RSU and the monitored vehicle, that is suspected by its neighbors as malicious. Each player chooses to perform a specific action in order to maximize its payoff. $\mathrm{J}_{\mathrm{RSU}}$ and $J_{v}$ denote respectively the RSU and the monitored vehicle players in the following. The RSU can perform one of these two pure strategies: detect, and categorize the monitored vehicle into a selected list (see subsection IV.C) or wait. The vehicle can attack or wait. At each state, our game represents an interaction between the RSU and vehicles located within its radio range. Here, we divide time into regular intervals called time-slots; each one of them represents a State. Therefore, with the help of Bayesian game concept, we model the different strategies that could occur between an attacker and the RSU, and the futures states' prediction of a suspected behavior is determined thanks to Bayesian Nash Equilibrium $(B N E)$.

In the following, we present the payoff matrix of the game between the monitored vehicle and RSU (see Table I), where some notations are defined as follows:

$-n b_{-}$false detection $_{\mathrm{i}, \mathrm{j}}$ : the rate of attackers $v_{j}$ that $R S U_{i}$ does not detect, i.e. false negative rate,

$-n b_{-}$false positive $i_{i, j}$ : the rate of normal vehicles $v_{j}$ that are detected by $R S U_{i}$ as malicious, i.e. false positive rate,

- nb_attacks detection $n_{i, j}$ : the rate of attackers $v_{j}$ that $R S U_{i}$ detects,

$-n b_{-}$forwarder : the rate of legitimate vehicles that accept and forward the false alert messages from a malicious vehicle,

- Cost: the percentage of overhead that RSU needs to detect and categorize the monitored vehicle into the appropriate list. 
$-\mathrm{P}=(\mathrm{p}, 1-\mathrm{p})$, where $\mathrm{p}$ and 1-p are the probabilities with which the RSU player performs a detect \& categorize or wait actions, respectively,

$-\mathrm{Q}=(\mathrm{q}, 1-\mathrm{q})$, where $\mathrm{q}$ and $1-\mathrm{q}$ are the probabilities with which the attacker player performs an attack or wait (i.e. don't attack) actions, respectively.

TABLE I. PAYOFF MATRIX OF INTRUSION DETECTION GAME

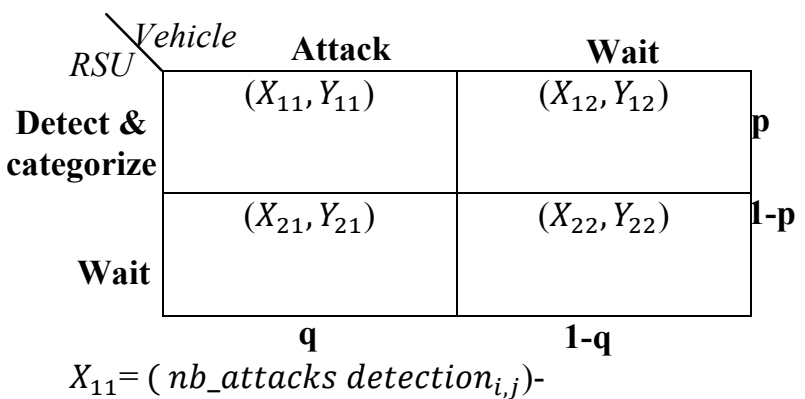

$\left(\right.$ Cost $+n b_{-}$false detection $\left._{\mathrm{i}, \mathrm{j}}\right)$

$$
\begin{aligned}
& X_{12}=-\left(\text { nb_false positive }_{\mathrm{i}, \mathrm{j}}+\mathrm{Cost}\right) \\
& X_{21}=-n b_{-} \text {false detection }_{\mathrm{i}, \mathrm{j}} \\
& X_{22}=0 \\
& Y_{11}=\left(\text { nb_false detection }_{i, j}+\text { nb_forwarder }_{j}\right)- \\
& n b_{\text {attacks detection }} \text { - }
\end{aligned}
$$

$$
\begin{aligned}
& Y_{12}=n b_{-} \text {false positive }_{\mathrm{i}, \mathrm{j}} \\
& Y_{21}=n b_{-} \text {false detection }_{i, j}+n b_{-} \text {forwarder }_{j} \\
& Y_{22}=0
\end{aligned}
$$

\section{B. Malicious behavior's prediction}

The utility of Bayesian Nash Equilibrium (BNE) is to predict the future behavior of a target vehicle since it aims to analyze the interaction between the attacker and the RSU, and determine the permanent state, i.e. each player has an interest in performing the same action. According to Nash, there is mixed strategy BNE player $_{l}\left(\right.$ action $\left._{1}, p^{*}\right)$, player $_{2}$ $\left(\right.$ action $\left.\left._{2}, q^{*}\right)\right\}$ in which both players do not change their actions. As a result, we use the BNE concept to predict the sate when the malicious vehicle persists to attack (i.e. does not switch to a normal behavior).

Theorem 1 There is a mixed strategy BNE $\left\{j_{R S U}\right.$ (detect and categorize, $\left.p^{*}\right), j_{v}($ attack, $\left.q *)\right\}$ in which the malicious vehicle attacks when the probability $q>q^{*}$ and the RSU triggers its detection (categorization) action when $p<p^{*}$.

Proof The mixed strategy of the RSU is defined as follows: $P=(p, 1-p)$, and the expected payoff of the malicious vehicle for playing attack and wait actions are:

$$
\begin{aligned}
& U_{J_{v}}(\operatorname{attack})=Y_{11} \cdot \mathrm{p}+Y_{21} \cdot(1-\mathrm{p}) \\
& =\left(n b \_ \text {false detection }_{i, j}+\text { nb_forwarder }_{j}\right)- \\
& n_{\text {n_attacks detection }}, j \cdot \mathrm{p} \\
& \begin{array}{l}
U_{J_{v}}(\text { wait })=Y_{12} \cdot \mathrm{p}+Y_{22} \cdot(1-\mathrm{p}) \\
=n b_{-} \text {false positive }{ }_{i, j} \cdot \mathrm{p}
\end{array}
\end{aligned}
$$

The malicious vehicle plays an attack action when $U_{J_{v}}($ attack $)>U_{J_{v}}($ wait $)$. Therefore, we get:

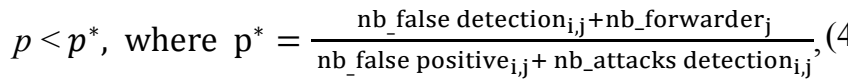

$$
\begin{aligned}
& \text { and } 0<p^{*} \leq 1
\end{aligned}
$$

The mixed strategy of a malicious vehicle is defined as follows: $Q=(q, 1-q)$ and the expected payoff of the RSU for playing detect (categorize) and wait actions are:

$$
\begin{aligned}
& U_{J_{R S U}}(\text { detect } \text { and categorize })=X_{11} \cdot \mathrm{q}+X_{12} \cdot(1-\mathrm{q}) \\
= & \left(\text { nb_attacks detection } n_{i, j}-n b_{-} \text {false detection }_{\mathrm{i}, \mathrm{j}}+\right. \\
\left.n b_{-} \text {false positive }_{\mathrm{i}, \mathrm{j}}\right) \cdot \mathrm{q}-\text { pb_false }_{-} \text {positive }_{\mathrm{i}, \mathrm{j}}-\text { Cost } & \\
& U_{J_{R S U}}\left(\text { wait }=X_{21} \cdot \mathrm{q}+X_{22} \cdot(1-\mathrm{q})\right. \\
=- & n b_{-} \text {false detection }_{\mathrm{i}, \mathrm{j}} \cdot \mathrm{q}
\end{aligned}
$$

The RSU plays the Detect \& categorize action when $U_{J_{R S U}}($ detect \& categorize $)>U_{J_{R S U}}$ (wait). Therefore, we can get:

$$
\begin{aligned}
& q>q^{*}, \text { where } q^{*}=\frac{\mathrm{nb}_{-} \text {false positive } \mathrm{e}_{\mathrm{i}, \mathrm{j}}+\text { Cost }}{\text { nb_attacks detection }_{i, j}+\text { nb false positive }_{\mathrm{i}, \mathrm{j}}},(5) \\
& \text { and } 0<q^{*} \leq 1
\end{aligned}
$$

As a result, we conclude that when the attack's probability of a malicious vehicle is above $q^{*}$ and the detection's probability of RSU is lower than $p^{*}$, both players do not change their actions. Therefore, when this equilibrium is reached, such malicious vehicle is detected as an attacker that persists to attack on the future states (which represent a Permanent state) and hence stored in the Revocation_Black list (as explained bellow).

\section{Monitored vehicules'categorization}

It is not interesting to remove the vehicle directly when it exhibits a malicious behavior since it could switch to a normal behavior and keeps this pattern during its passage through a network [5]. Thereby, the RSU categorizes the monitored vehicles into four lists:

- White list. The vehicle that acts as normal node during its passage through RSU's range, its $M P$ is equal to 0 .

- White \& Gray list. The vehicle oscillates between a normal and malicious behavior. However, in the future states, the rate of switching to malicious behavior is less than to switching in a normal behavior, $q 1^{*} \leq M P<q 2^{*}$; such vehicle is defined as Abnormal node.

- Gray list. The vehicle oscillates between a normal and malicious behavior. However, in the future states the rate of switching to malicious behavior is more than to switching in a normal behavior, $q 2^{*} \leq M P$; such vehicle is defined as Suspect node.

- Revocation_Black list. The vehicle oscillates between a normal and malicious behavior. However, in the future states it persists to act as malicious node, i.e. does not switch on a normal behavior. In other word, its $M P$ converges to 1 . Here $M P>q^{*}$ (Nash equilibrium). 
Here, $q^{*}>q 2^{*}>q 1^{*}$. The probability $q^{*}$ is determined according to Nash equilibrium concept (see equation 5). However, the optimal values of the probabilities $q 1^{*}$ and $q 2^{*}$ are determined in our experiment which should satisfy our application requirement, i.e. high accuracy prediction. We note that, the vehicles that are stored in a Revocation Black list will be totally prevented to communicate with legitimate vehicles. As cited above, RSUs exchange this list and each RSU informs the vehicles located within its radio range about the identities of these nodes.

\section{PERFormance EVAluation}

Our approach was implemented in NS-3.17 (Network Simulator) [12]. In this section, first of all we study the optimal probabilities thresholds $q 1 *$ and $q 2 *$ that allow a high accuracy prediction, i.e. high detection and low false positive rates. Afterward, we compare our intrusion detection and prevention schema with two detection frameworks: Intrusion Detection Framework for Vehicular Network (IDFV) [2] and Data-Centric Misbehavior Detection (DCMD)[5]. Here, we evaluate the prediction performance; in other words the accuracy prediction (i.e. detection and false positive rates) of the future behavior of a monitored vehicle and its categorization in an appropriate list. Finally, we evaluate the overhead generated by our schema. These metrics are defined here after.

- Detection Rate (DR): ratio of correctly identified malicious vehicles and their categorization in appropriate lists,

- False Positive Rate (FPR): ratio between the number of normal vehicles that are incorrectly classified as malicious,

- Communication Overhead (COV): the amount of information generated by the vehicle.

These first two metrics allow us to evaluate the accuracy prediction. The last one is a network performance metric. In our simulation, we used two topologies: two parallel highways (2x3 lanes) and urban scenario generated by the Simulation of Urban Mobility (SUMO) simulator. Furthermore, we simulated a square area of $3000 \times 3000 \mathrm{~m}^{2}$. Our measurements are based on averaging the results obtained from 15 simulation runs. Moreover, in our simulation, we vary the number of malicious vehicles from 10 to $40 \%$ of overall nodes. We insert, three categories of attackers: abnormal node, suspect node and malicious node that persist to attack, which are stored in a White \& Gray, Gray and Revocation_Black lists, respectively. The main simulation parameters are summarized in Table II. These parameters were chosen to be as most realistic as possible.

TABLE II. SIMULATION PARAMETERS

\begin{tabular}{|c|c|}
\hline Simulation area & $3000 \times 3000 \mathrm{~m}^{2}$ \\
\hline Simulation time & 180 seconds \\
\hline $802.11 \mathrm{p}$ maximum range & 300 meters \\
\hline Vehicles number & From 50 to 250 \\
\hline Vehicles velocity & 90 to $160 \mathrm{~km} / \mathrm{h}$, step 15 \\
\hline Detection Period $(\Delta \mathrm{t})$ & 7 seconds \\
\hline
\end{tabular}

\section{A. Optimal probabilities thresholds}

The identification of the optimal probabilities $q 1^{*}$ (that categorizes the monitored vehicles into White \& Gray list) and $q 2 *$ (related to the monitored vehicles that are stored in a Gray list) is crucial. In one hand, when these values are low, the number of false positives is important. In the other hand, when these thresholds are high, a great number of malicious vehicles are not detected. Therefore, a tradeoff between detection and false positive rates should be considered. The probabilities thresholds are computed as follow:

$$
\begin{aligned}
& \mathrm{q} 1 *=\mathrm{q}^{*}-\delta \\
& \mathrm{q} 2^{*}=\mathrm{q}^{*}-\gamma
\end{aligned}
$$

We varied the values $\delta$ and $\gamma$, afterward we compute the detection and false positive rates for low and high number of malicious vehicles $(10 \%, 20 \%, 30 \%$ and $40 \%$ of overall nodes). Therefore, the optimal probabilities thresholds that make a balance between detection and false positive rates are selected. We note that, $q$ * is determined according to the BNE concept (see equation 5).

TABLE III. OPTIMAL THRESHOLDS

\begin{tabular}{|c|l|c|c|c|}
\hline $\begin{array}{c}\text { Number of attackers } \\
(\%)\end{array}$ & $\boldsymbol{\delta}\left(\mathrm{q} 1^{*}\right)$ & $\gamma\left(\mathrm{q} 2^{*}\right)$ & DR (\%) & FPR(\%) \\
\hline 10 & 0,42 & 0,28 & 100 & 0 \\
\hline 20 & 0,45 & 0,29 & 98 & 0,5 \\
\hline 30 & 0,49 & 0,34 & 97,33 & 1,15 \\
\hline 40 & 0,56 & 0,37 & 97 & 1,35 \\
\hline
\end{tabular}

According to our simulation results, we found that there are optimal probabilities thresholds $\left(q^{*}, q 1^{*}\right.$ and $\left.q 2^{*}\right)$ that allow us to detect and predict with a high accuracy the future malicious behavior of a monitored vehicle and hence categorize it in an appropriate list. Furthermore, the optimal values of $\delta$ and $\gamma$ that make a balance between detection and false positive rates are illustrated in Table III.

\section{B. Performance comparison}

In this subsection, we first compare the performance of our schema with IDFV [2] and DCMD [5] in terms of detection and false positive rates. Both frameworks attempt to detect the current attacks that occur on network, where the first one aims to detect the DoS attack and a second one the false alert's generation attack. Afterward, we analyze the overhead generated by our schema. We vary the number of vehicles from 50 to 250 . We note that, we use the optimal probabilities thresholds $\left(\delta\left(q 1^{*}\right)=0,56\right.$ and $\left.\gamma\left(q 2^{*}\right)=0,37\right)$ found above and fix the number of malicious vehicles equal to $40 \%$ (maximum, worst case) of overall nodes. We fix the maximum number of malicious nodes equal to $40 \%$ of overall nodes as when this ratio exceeds this value, the performances of our schema degrade significantly (i.e. high FPR and low DR).

\section{1) Accuracy prediction}

According to Fig. 3 (a) and (b), we show that our schema exhibits a high accuracy prediction compared to IDFV and DCMD since the detection rate is over $98 \%$ and the false positive rate is lower than $2 \%$ when the number of vehicles 
increases, which demonstrate that our schema is scalable. This results is achieved thanks to the malicious behavior's prediction concept that has the ability to predict with a high accuracy future misbehavers and categorize them in appropriate list. This is unlike IDFV and DCMD frameworks, where there is no prevention technique since they aim to detect only current attackers that occur in the network.

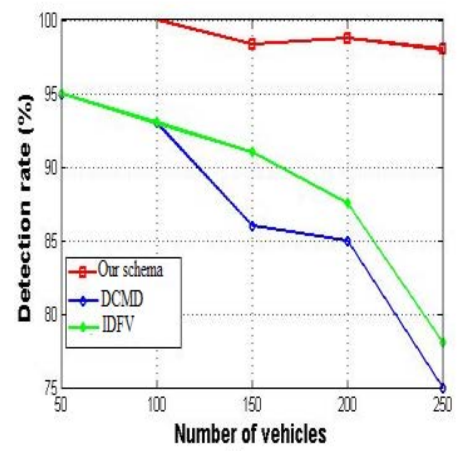

(a)

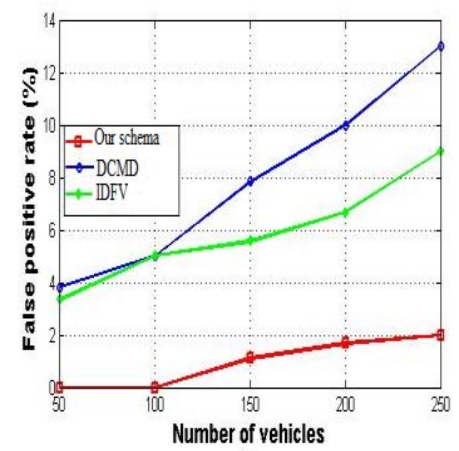

(b)
Fig. 3. Accuracy prediction: (a) Detection rate and (b) False positive rate

\section{2) Overhead}

In this research, we propose a hybrid intrusion detection and decision schema, where the detection is performed in a distributed manner between vehicles and the decision is performed by a centralized RSU. We compare our hybrid schema with a distributed one in terms of overhead. As illustrated in Fig 4 , both schema generate the same amount of overhead when the number of vehicles is equal to 50 . However, when this number increases, the distributed schema generates a high overhead compared to hybrid one. This result is achieved since in a distributed schema, each vehicle exchanges with its neighbors the malicious lists (described above) in order to propagate them to all nodes within a network. Therefore, such schema leads to an increase on overhead, specifically when the number of malicious vehicles is important. Furthermore, in a hybrid schema only the RSU disseminates the complete lists to the vehicles (located within it radio range) since it has the lists of all malicious vehicles that are detected within a network.

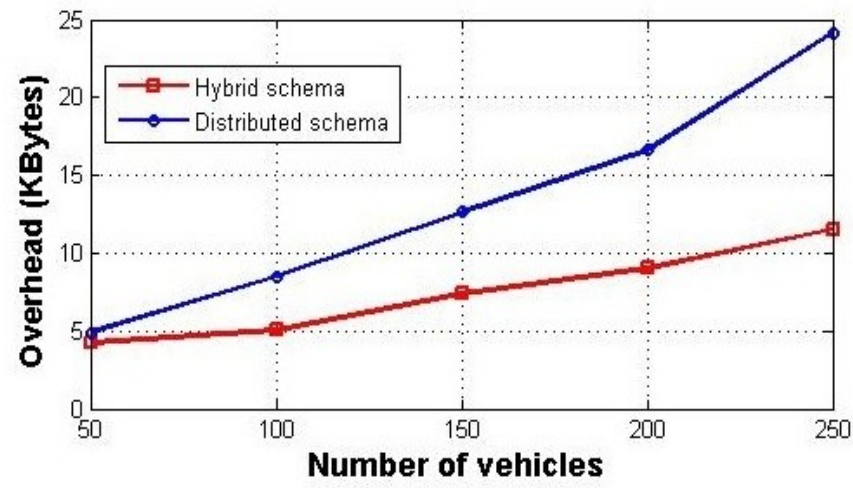

Fig. 4. Communication overhead

\section{CONCLUSION}

In this paper, we propose and design a lightweight and efficient intrusion detection and prevention schema for vehicular networks. To point out, with a help of game theory, our schema has the ability to predict with a high accuracy a future malicious behavior of an attacker and categorize it into an appropriate list according to the future attack severity. According to our simulation results, we demonstrate that our intrusion detection and prevention schema exhibits a high detection rate i.e. over $98 \%$, low false positive rate, i.e. close $2 \%$ and generates a low overhead when the number of malicious vehicles is equal to $40 \%$ (maximum, worst case) of overall nodes. This result is achieved, when a high number of vehicles is deployed (250 nodes). Our intrusion detection and prevention schema is flexible and could be embedded either on a flat or a hierarchical topology. Therefore, in near future we will embed our schema in a cluster-based topology and evaluate these both topologies in term of generated overhead.

\section{ACKNOWDELGMENT} Code.

This work has been funded by the European project Car-

\section{REFERENCES}

[1] ITEA 2 CarCoDe project (2013-2015), http://www.itea2-carcode.org/

[2] H. Sedjelmaci, and SM. Senouci, "A New Intrusion Detection Framework for Vehicular Networks", IEEE ICC, Sydney, Australia, 1014 June 2014.

[3] H. Sedjelmaci, S.M. Senouci, and M. Feham, "An efficient intrusion detection framework in cluster-based wireless sensor networks", Security and Communication Networks , 2013, pp. 1211-1224.

[4] T. Gazdar, A. Rachedi, A. Benslimane, and A. Belghith. "A Distributed Advanced Analytical Trust Model for VANETs", IEEE GLOBECOM, California, USA, 2012, pp. 201-206.

[5] S. Ruj, M.A. Cavenaghi, Z. Huang, A. Nayak, and I. Stojmenovic, "On Data-centric Misbehavior Detection in VANETs", IEEE Vehicular Technology Conference (VTC Fall), San Francisco, USA, 2011, pp. 15.

[6] F. Marmol, G. Perez, TRIP: a trust and reputation infrastructure-based proposal for vehicular ad hoc networks, Journal of Network and Computer Applications, 35(3), 2011, pp.934-941.

[7] F. Marmol, G. Perez, TRIP: a trust and reputation infrastructure-based proposal for vehicular ad hoc networks, Journal of Network and Computer Applications, 35(3), 2011, pp.934-941.

[8] A. Daeinabi, A. G. P. Rahbar, and A. Khademzadeh, "VWCA: An efficient clustering algorithm in vehicular ad hoc networks", Journal of Network and Computer Applications, 34 (1), 2011, pp. 207-222.

[9] C. Zhang, R. Lu, X. Lin, P.H. Ho, and X. ShenAn, "Efficient Identitybased Batch Verification Scheme for Vehicular Sensor Networks", IEEE INFOCOM proceeding, Phoenix, USA, 2008, pp.816-824.

[10] H. Zhu, R. Lu, X. Lin, and Xuemin. Shen, "Security in Service-Oriented Vehicular Networks", IEEE Wireless Communications, vol. 16, no.4, pp. 16-22, August, 2009.

[11] Z.Md. Fadlullah, T. Taleb, A. V. Vasilakos, M. Guizani, and N. Kato: "DTRAB: Combating Against Attacks on Encrypted Protocols Through Traffic-Feature Analysis", IEEE/ACM Trans. Netw. 18(4), 2010, pp. 1234-1247.

[12] http://www.nsnam.org. 